\title{
Balanitis Xerotica Obliterans
}

National Cancer Institute

\section{Source}

National Cancer Institute. Balanitis Xerotica Obliterans. NCI Thesaurus. Code C3523.

A chronic and progressive inflammatory process that affects the glans penis and the foreskin. It presents with white atrophic patches in the glans of penis and foreskin and it is often associated with the development of a sclerotic, whitish ring in the tip of the foreskin that may lead to phimosis. It is also known as lichen sclerosus of the penis. 